\title{
Seismic analysis of the planet-hosting star $\mu$ Arae
}

\author{
M. Bazot ${ }^{1}$, S. Vauclair ${ }^{1}$, F. Bouchy ${ }^{2,3}$, and N. C. Santos ${ }^{4,5}$ \\ ${ }^{1}$ Laboratoire d'Astrophysique de l'Observatoire Midi-Pyrénées, CNRS, UMR 5572, UPS, 14 Av. E. Belin, \\ 31400 Toulouse, France \\ e-mail: bazot@ast.obs-mip.fr \\ 2 Laboratoire d'Astrophysique de Marseille, Traverse du Siphon, 13013 Marseille, France \\ 3 Observatoire de Haute-Provence, 04870 Saint Michel l'Observatoire, France \\ ${ }^{4}$ Lisbon Observatory, Tapada da Ajuda, 1349-018 Lisboa, Portugal \\ 5 Observatoire de Genève, 51 chemin des Maillettes, 1290 Sauverny, Switzerland
}

Received 13 January 2005 / Accepted 5 March 2005

\begin{abstract}
Like most exoplanet host stars, HD 160691 (alias $\mu$ Ara) presents a metallicity excess in its spectrum compared to stars without detected planets. This excess may be primordial, in which case the star would be completely overmetallic, or it may be due to accretion in the early phases of planetary formation, in which case it would be overmetallic only in its outer layers. As discussed in a previous paper, seismology can help in choosing between the two scenarios. This star was observed during eight nights with the spectrograph HARPS at La Silla Observatory. Forty three $p$-modes were previously identified. In the present paper, we discuss the modelling of this star. We computed stellar models iterated to present the same observable parameters (luminosity, effective temperature, outer chemical composition) with differing internal structure according to the two extreme assumptions: original overmetallicity or accretion. We show that the seismic constraints lead to models in agreement with the external parameters deduced from spectroscopy and from the Hipparcos parallax ( $L$ and $T_{\text {eff }}$ ). We show that the "small separation" seems to give a better fit for the accretion case than for the overmetallic case, but in spite of the very good data the uncertainties are still too large to be conclusive. We discuss the observations needed to resolve this question.
\end{abstract}

Key words. stars: oscillations - stars: abundances - stars: planetary systems: formation stars: supernovae individual: HD 160691

\section{Introduction}

Since the first discovery of a planet orbiting around Peg 51 (Mayor \& Queloz 1995), more than 130 exoplanets have been detected ${ }^{1}$. The central stars of these planetary systems appear to be overmetallic compared to the Sun, at least in their atmospheres. Their average metallicity is $\sim 0.2$ dex larger than solar while it is about solar for stars with no detected planets, in the solar neighborhood (Gonzalez 1998; Santos et al. 2003). Two scenarios have been proposed to explain these high metallicities. In the first scenario, they are the result of a high initial metal content in the proto-stellar gas: in a metal-rich environment the mechanisms leading to planet formation are more efficient, which would explain the observed bias. In the second scenario, the overmetallicity is due to the accretion of hydrogen-poor matter during planet formation. This matter could come from already formed planets that migrate towards the central star (Trilling et al. 1998) and eventually fall onto it (Murray et al. 2001; Murray \& Chaboyer 2002). This process leads to a metal excess in the stellar outer layers only (as it is diluted in the surface convective zone), while the former

${ }^{1}$ See for example http://www . obspm.fr/encycl/cat 1. html scenario implies that planet-hosting stars are completely overmetallic, down to their centers.

Bazot \& Vauclair (2004, hereafter BV04) studied the internal differences of stellar models computed with either the overmetallic or the accretion assumption, but iterated so as to present exactly the same observable parameters $\left(T_{\text {eff }}, \frac{L}{L_{\odot}}, Z\right)$ : these models can account for the same observed star, but their interiors are different. Moreover, they have slightly different masses (up to 20\%) while their observed parameters are the same. BV04 studied the asteroseismic signatures of these internal differences. They focused on the fact that a metal-dependent process occurs in stars with masses around $1.1 M_{\odot}$, as convective cores begin to develop (Michaud et al. 2004). Thus, it is possible that among two models representing the same observed star, one has a convective core while the other does not.

We test these preliminary investigations with the star $\mu$ Ara (HD 160691, HR 6585, GJ 691). We first reported a discovery of a new low-mass planet around this star, of $\sim 14$ earth masses (Santos et al. 2004b). The star itself presents very clear oscillations: the analysis of the Fourier transform of the radial velocity time series allowed us to detect 43 oscillation modes of degrees $l=0$ to $l=3$ (Bouchy et al. 2005, hereafter Paper I). 
A summary of these observations is given in Sect. 2. The model computations and comparison with the observations are presented in Sect. 3. The results are discussed in Sect. 4 while we conclude and propose future work in Sect. 5.

\section{Observational constraints}

With a visual magnitude of $V=5.1$ and a Hipparcos parallax of $\pi=65.5 \pm 0.8$ mas, the star $\mu$ Ara has an absolute magnitude $M_{V}=4.20$ and a luminosity of $\log L / L_{\odot}=0.28 \pm 0.012$. The different values given in the literature for its effective temperature are listed in Table 1. The HARPS spectrum obtained during our run (see below) was used to derive a new spectroscopic set of atmospheric parameters. From this study the values $T_{\text {eff }}=5813 \pm 40 \mathrm{~K}$ and $[\mathrm{Fe} / \mathrm{H}]=0.32 \pm 0.05$ dex were found (second line of Table 1). With such a metallicity, $\mu$ Ara is one of the most metallic planet-hosting star (see e.g. Santos et al. 2003). In Table 1 there is a discrepancy in effective temperature between the two oldest measurements (bottom lines) and the most recent ones which are in good agreement, around 5800 K. In Figs. 1 and 2, the five most recent measurements are given. We will see below that seismic studies of this star lead to more precise constraints on $T_{\text {eff }}$, inside the error bars given by these spectroscopic values. They are in good agreement with the newest effective temperatures, wh ich are believed to be more precise than the oldest ones. The two lowest values given in Table 1 for $T_{\text {eff }}$ are ruled out by our results.

We observed $\mu$ Ara with the high-precision echelle spectrograph HARPS on the 3.6-m ESO telescope at La Silla, Chile. As discussed in detail in Paper I, 43 low degree $p$-modes with a signal-to-noise ratio greater than 2.5 were identified. The degrees of the observed modes range from $l=0$ to $l=3$. The uncertainty on each mode frequency is $0.78 \mu \mathrm{Hz}$ due to the duration of the time series. The eigenfrequencies for these $p$-modes are given in Table 2 of Paper I. The average separation between modes of consecutive order is $\Delta v_{0}=90 \mu \mathrm{Hz}$ (see Paper I). Because of the different confidence levels on the observed frequencies (see paper I for a complete discussion), and thus on the individual large separations, we defined the region of seismic constraint as the area delimited by the iso- $\Delta v_{0} 89 \mu \mathrm{Hz}$ and $91 \mu \mathrm{Hz}$ (see Figs. 1 and 2).

\section{Models}

\subsection{Echelle diagrams}

We computed series of "overmetallic" and "accretion" models which could account for the observed parameters of $\mu$ Ara. We used the Toulouse-Geneva stellar evolution code, with the OPAL equation of state and opacities (Rogers \& Nayfonov 2002; Iglesias \& Rogers 1996) and the NACRE nuclear reaction rates (Angulo et al. 1999). In all our models, microscopic diffusion was included using the Paquette prescription (Paquette et al. 1986; Richard et al. 2004). The treatment of convection was done in the framework of the mixing length theory and the mixing length parameter was adjusted as in the Sun ( $\alpha=1.8$, see Richard et al. 2004). The accretion models were computed with the same assumptions as in BV04
Table 1. Effective temperatures and metal abundances observed for $\mu$ Ara. $[\mathrm{Fe} / \mathrm{H}]$ ratios are given in dex. The references for the given values are given in Col. 3; they are followed by $\dagger$ for spectrometric measurements and $\ddagger$ for photometric measurements.

\begin{tabular}{lcc}
\hline \hline \multicolumn{1}{c}{$T_{\text {eff }}(K)$} & {$[\mathrm{Fe} / \mathrm{H}]$} & Reference \\
\hline $5798 \pm 33$ & $0.32 \pm 0.04$ & Santos et al. (2004a) $\dagger$ \\
$5813 \pm 40$ & $0.32 \pm 0.05$ & Santos et al. (2004b) $\dagger$ \\
$5800 \pm 100$ & $0.32 \pm 0.10$ & Bensby et al. (2003) $\dagger$ \\
$5811 \pm 45$ & $0.28 \pm 0.03$ & Laws et al. (2003) $\dagger$ \\
$5800 \pm 90$ & $0.28 \pm 0.04$ & Favata et al. (1997) $\dagger$ \\
$5570 \pm 70$ & $0.39 \pm 0.10$ & Laird et al. (1985) $\ddagger$ \\
$5597 \pm 160$ & $0.41 \pm 0.15$ & Perrin et al. (1977) $\dagger$ \\
\hline
\end{tabular}

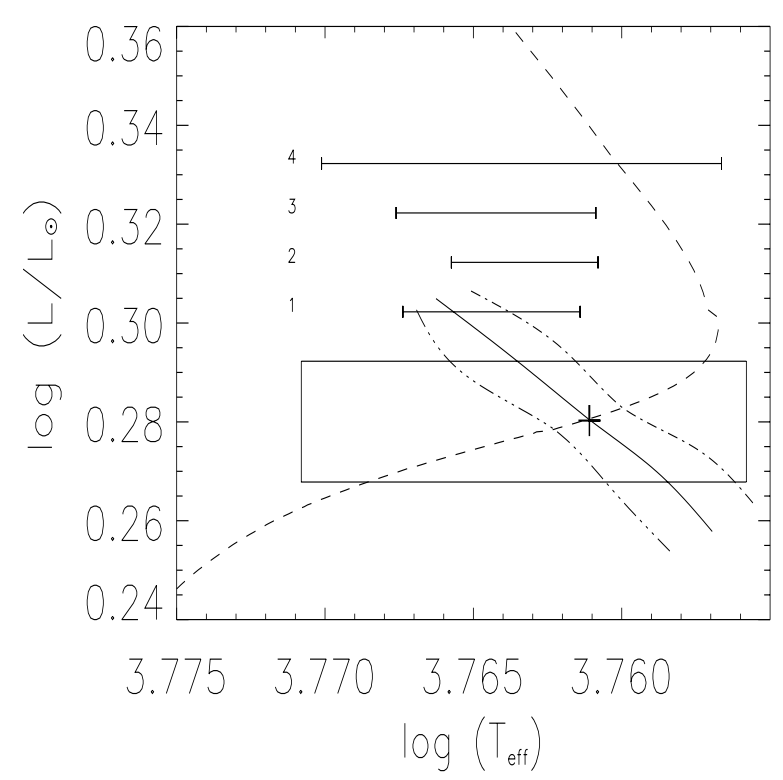

Fig. 1. Constraints on the position of the star $\mu$ Ara in the HR diagram. The error box corresponds to the luminosity derived from the Hipparcos parallax and the effective temperature measured by Bensby et al. (2003). Other temperature determinations are: 1 - Santos et al. (2004a), 2 - Santos et al. (2004b), 3 - Laws et al. (2003) and 4 - Favata et al. (1997). An example of an evolutionary track is drawn for the "overmetallic" assumption (dashed line, see text). The asteroseismic constraints appear as three oblique lines: center line (solid) represents the models for which the large separations are exactly $90 \mu \mathrm{Hz}$ as observed in $\mu$ Ara, while the two other lines stand for $89 \mu \mathrm{Hz}$ (top dot-dashed line) and $91 \mu \mathrm{Hz}$ (bottom dot-dashed line). The cross shows the point discussed in more detail, for comparison with the accretion case.

(instantaneous infall of matter at the beginning of the main sequence and instantaneous mixing inside the convection zone). Neither extra-mixing nor overshoot were taken in account in the present computations (this will be done in future work).

Adiabatic oscillations frequencies were computed for a large number of models along the evolutionary tracks, using a recent version of the Brassard et al. (1992) code, as in BV04. In order to compare them with the modes observed in $\mu$ Ara, the frequencies were computed for angular degrees $l=0$ to $l=3$ and radial orders ranging typically from 4 to 100 . The azimuthal order is always $m=0$. For each evolutionary track, we focused on the models which fell inside the observed box in the 


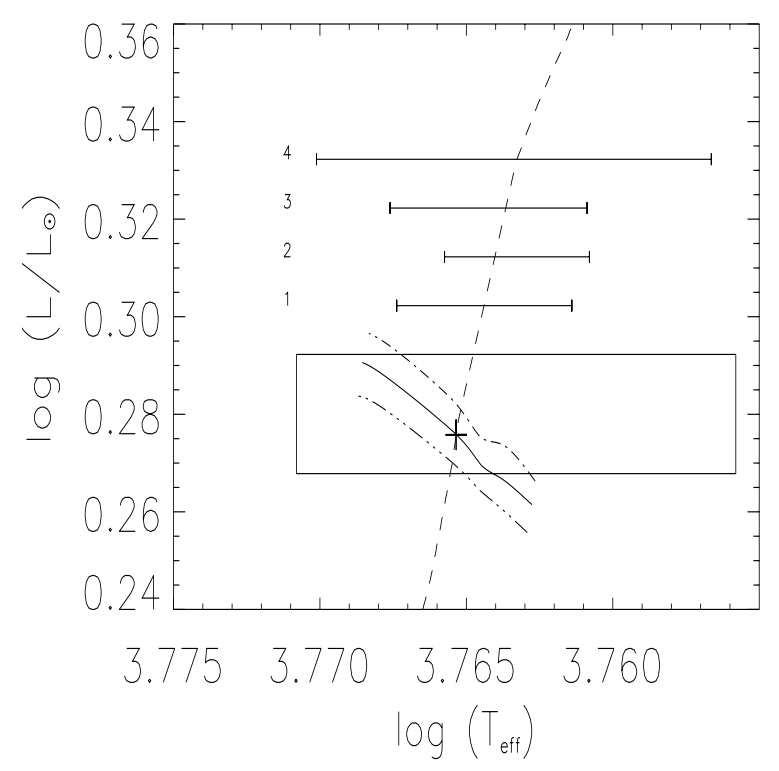

Fig. 2. Same as Fig. 1 for the observational constraints on $\mu$ Ara. Here the evolutionary track is drawn for the "accretion" assumption. The seismic constraints are represented for the accretion case as in Fig. 1.

HR diagram (cf. Figs. 1 and 2), namely models with luminosities and effective temperatures as given by spectroscopy. We then searched for those models that could precisely reproduce the observed echelle diagram.

For each track, only models with computed large separations of exactly $90 \mu \mathrm{Hz}$ could fit the observed echelle diagram. A difference of only $0.5 \mu \mathrm{Hz}$ in the large separations destroys the agreement. The oblique solid lines in Figs. 1 and 2 represent the location in the HR diagram of the models that correctly fit the echelle diagram and lie in the spectroscopic error box. The two other curves (dot-dashed ones) indicate models for which the large separations are respectively 89 and $91 \mu \mathrm{Hz}$, which no longer fit the echelle diagram.

We present here two models which, given our basic assumptions, fit reasonably well the observed frequencies and echelle diagram. We computed a reduced $\chi^{2}$ for both cases. The echelle diagrams are given in Figs. 3 and 4, respectively for the overmetallic and the accretion cases. These models correspond to the crosses in Figs. 1 and 2. Their characteristics are given in Table 2. The accretion model is labeled AC and the overmetallic one OM. The internal distributions of elements in these models are displayed in Fig. 5: in the AC model the overmetallicity lies only in the outer layers while it is present in the whole OM model. The initial helium mass fraction $Y_{0}$ is larger for the overmetallic model than for the accretion model as we assume here that the original interstellar matter was overabundant in metals and helium according to the chemical evolution of galaxies (Izotov \& Thuan 2001, see BV04 for a discussion of this assumption). From spectroscopy and photometry both stars would appear nearly identical, within the observational uncertainties, but their internal structures are quite different. The most striking fact is the presence of a convective core in the overmetallic model, with a radius of $\sim 0.07 R_{\star}\left(R_{\star}\right.$ being the radius of the star).

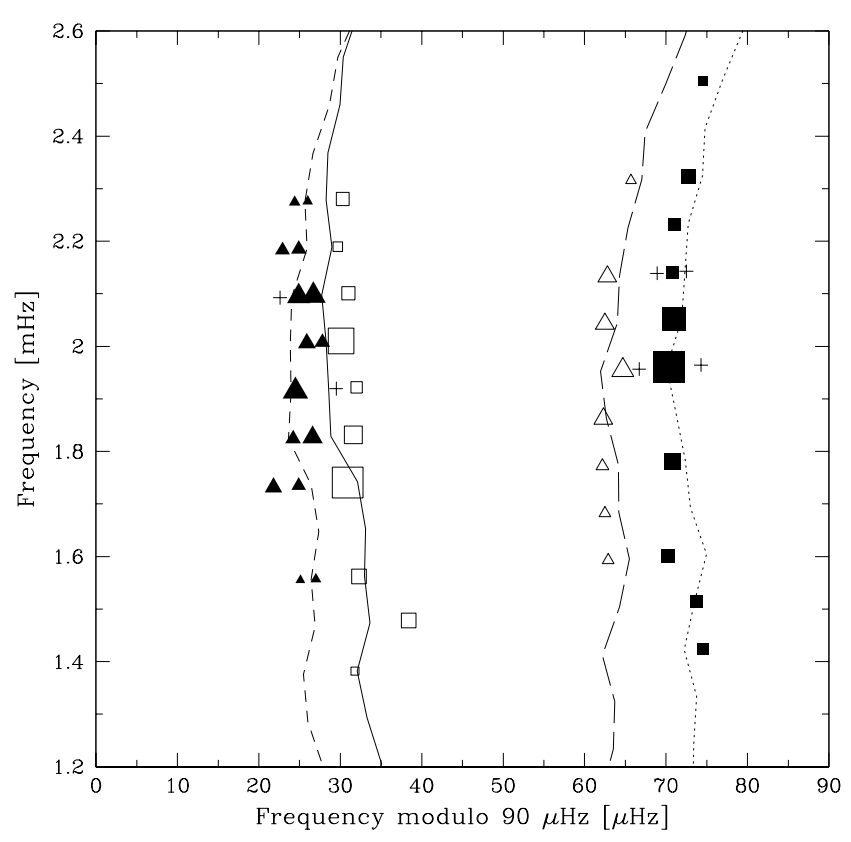

Fig. 3. Echelle diagram for the overmetallic model. The lines represent the theoretical frequencies, the solid line is for $l=0$, the dotted line for $l=1$, the dashed line for $l=2$ and the long-dashed line for $l=3$. The symbols represent their observational counterpart. Open squares, full squares, open triangles and full triangles stand for $l=0, l=1$, $l=2, l=3$, respectively. Their size is proportionnal to the signal to noise ratio (see Paper I).

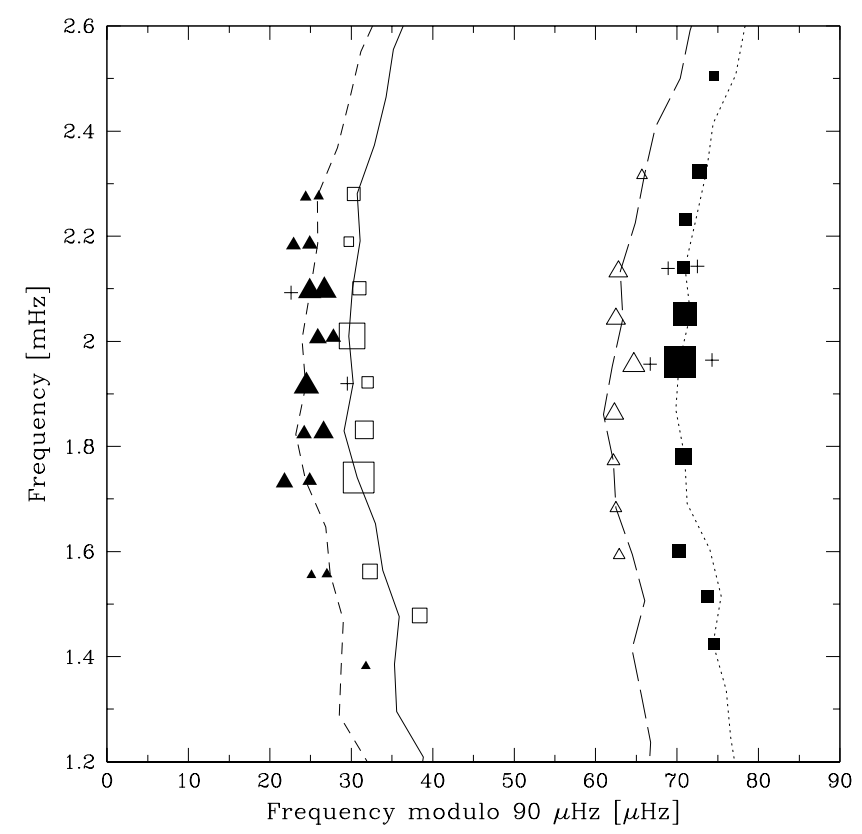

Fig. 4. Same as Fig. 3 for the accretion model. Only the mode with the lowest frequency differs from Fig. 3 (see text).

The accretion scenario, with $\chi 2=4.3$, provides a better match for the observations than the overmetallic model, for which we obtained $\chi 2=7.6$. These values are still high. However, we discuss below other possible seismic tests.

The lowest frequency has been identified as a $l=2$ mode (see Paper I). It is in good agreement with the accretion model. However, the overmetallic model gives a slightly better fit if 
Table 2. Mass, age, initial and actual chemical compositions, radii, geometrical and acoustic, of the star $\left(R_{\star}\right.$ and $\left.T_{\star}\right)$ and of the convective core $\left(R_{\mathrm{cc}}\right.$ and $t_{\mathrm{cc}}$, when necessary) for stellar models that satisfy the observational constraints for $\mu$ Ara. The model AC includes accretion, OM is overmetallic. Initial mass fractions $\left(X_{0}, Y_{0}, Z_{0}\right)$ on the main sequence are given for the core and the envelop, which has been enhanced by pollution in the $\mathrm{AC}$ case. For the final model, the surface abundances are given $(X, Y, Z)$.

\begin{tabular}{|c|c|c|c|c|c|c|c|c|c|c|}
\hline \multirow[t]{2}{*}{ Model } & \multirow{2}{*}{$\begin{array}{c}M_{\star} \\
\left(M_{\odot}\right)\end{array}$} & \multirow{2}{*}{$\begin{array}{c}\text { Age } \\
(\mathrm{Gyr})\end{array}$} & \multirow{2}{*}{$\begin{array}{l}T_{\text {eff }} \\
(\mathrm{K})\end{array}$} & \multirow[t]{2}{*}{ 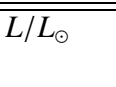 } & \multicolumn{2}{|c|}{$\overline{X_{0}}$} & \multicolumn{2}{|c|}{$Y_{0}$} & \multicolumn{2}{|c|}{$\overline{Z Z_{0}}$} \\
\hline & & & & & envel. & core & envel. & core & envel. & core \\
\hline $\mathrm{AC}$ & 1.085 & 5.803 & 5825.69 & 1.887 & 0.6913 & 0.7097 & 0.2650 & 0.2714 & 0.0437 & 0.0189 \\
\hline \multirow[t]{4}{*}{$\mathrm{OM}$} & 1.180 & 4.251 & 5769.28 & 1.908 & 0.6696 & 0.6696 & 0.2891 & 0.2891 & 0.0413 & 0.0413 \\
\hline & & $\overline{\bar{X}}$ & $\overline{\bar{Y}}$ & $\overline{\overline{Z Z}}$ & $\begin{array}{c}R_{\star} \\
\left(R_{\odot}\right) \\
\end{array}$ & $\begin{array}{l}R_{\mathrm{cc}} \\
\left(R_{\star}\right) \\
\end{array}$ & $\begin{array}{l}T_{\star} \\
(\mathrm{s})\end{array}$ & $\begin{array}{l}t_{\mathrm{cc}} \\
(\mathrm{s})\end{array}$ & & \\
\hline & & 0.7492 & 0.2124 & 0.0384 & 1.35 & - & 5258.54 & - & & \\
\hline & & 0.6694 & 0.2892 & 0.0414 & 1.38 & 0.07 & 5240.80 & 137.19 & & \\
\hline
\end{tabular}
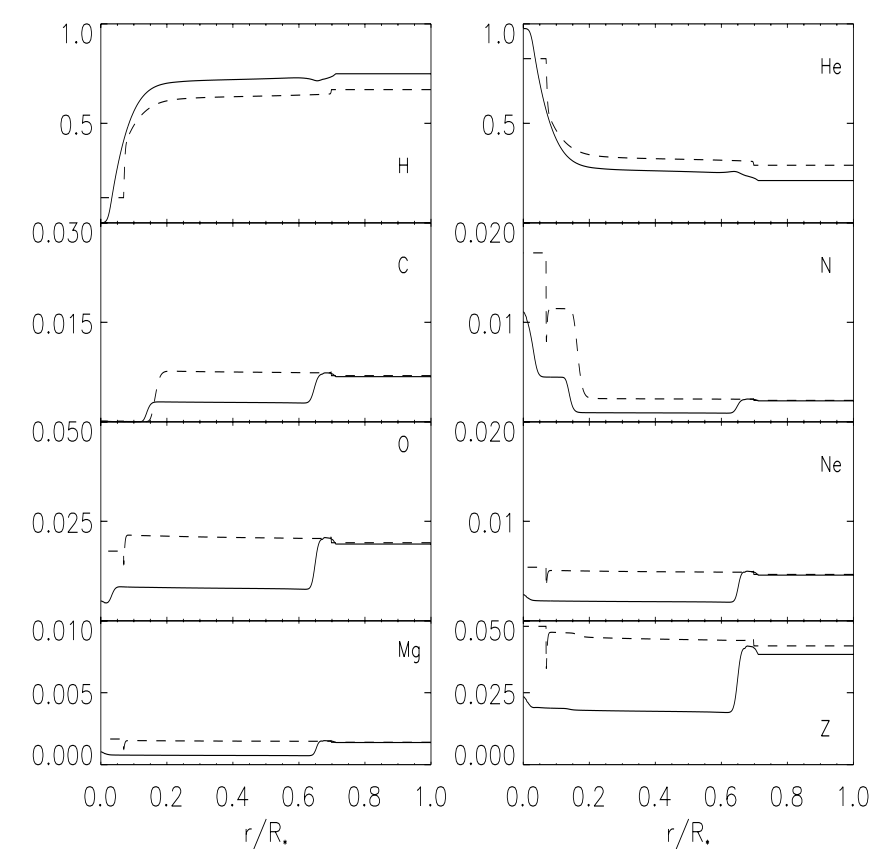

Fig. 5. Internal distribution for elements $\mathrm{H}, \mathrm{He}, \mathrm{C}, \mathrm{N}, \mathrm{O}, \mathrm{Ne}$ and $\mathrm{Mg}$ for models OM (dashed lines) and AC (full lines). The lower right panel displays the global fraction, $\mathrm{Z}$, of elements heavier than helium.

we consider it as being a $l=0$ mode. This is an example of the uncertainty on the mode identification at very low signal to noise ratios.

\subsection{Small separations}

We compared the measured small separations, $\delta v=\delta v_{n, l}-$ $v_{n-1, l+2}$, with the computed ones. As this quantity is very sensitive to the deep stellar interior (Tassoul 1980; Roxburgh \& Vorontsov 1994), it was proposed in BV04 as a good indicator of the presence of a convective core in stars around 1.1 $M_{\odot}$.

According to Roxburgh \& Vorontsov (1994), the presence of a convective core leads to an oscillatory signal in the small separations with a period scaling approximately as $\sim 1 / 2 t_{\mathrm{cc}}$, where $t_{\mathrm{cc}}$ is the acoustic radius of the convective core (see Table 2).

Figures 6 and 7 display the observed and computed small separations for the $l=0,2$ and $l=1,3$ cases. The overmetallic and the accretion cases are different for the $l=0,2$ small separations. In the overmetallic case they rapidly decrease at large frequencies and become negative, while in the accretion case the curve is much flatter. This behavior has to be related to the echelle diagrams: for the overmetallic case (Fig. 3), the theoretical lines for $l=0$ and $l=2$ cross around $v=2.7 \mathrm{mHz}$, which does not happen for the accretion case. Such a crossing is in contradiction to the asymptotic theory which may be a good representation of seismology in a radiative interior but does not hold in the presence of a convective core. It assumes that the sound velocities at the turning points of the waves are identical to the central sound velocity, which is not true in this case (Fig. 8). A complete discussion of the signatures of convective cores will be given in a forthcoming paper.

We also computed a reduced $\chi 2$ for the small separations. We obtained $\chi 2=1.2$ for model AC and $\chi 2=2.2$ for model OM. These results are better than those resulting from the fits of the frequencies (echelle diagrams). This is partly due to the fact that the uncertainties on small separations are $\sqrt{2}$ times larger than for individual frequencies. But there is also a physical reason for this better fit: the small separations are believed to be independent of the surface effects which affect the individual frequencies (see e.g. Gabriel 1989). These effects, which are difficult to estimate, may deteriorate the quality of the fit of individual frequencies while they have no influence on small separations.

That model AC better matches the observations than model OM should be considered cautiously. Figure 6 shows that the decrease of the theoretical small separations due to the convective core in model OM becomes more important at high frequencies: this effect, which is crucial for the choice between models, corresponds to the beginning of the oscillations that we have discussed above. However, the three values that we obtain for the highest observed frequencies involve modes with a signal to noise ratio between 2.5 and 3 (small dots in Figs. 6 and 7). We cannot exclude misidentifications of these modes. In such a case the corresponding small separations would be different and the agreement between the observations and model OM could become comparable to the one with model AC.

More observations are needed to reach a clear conclusion. It would be particularly important to detect modes with large frequencies, even larger than in the present work, and with the 


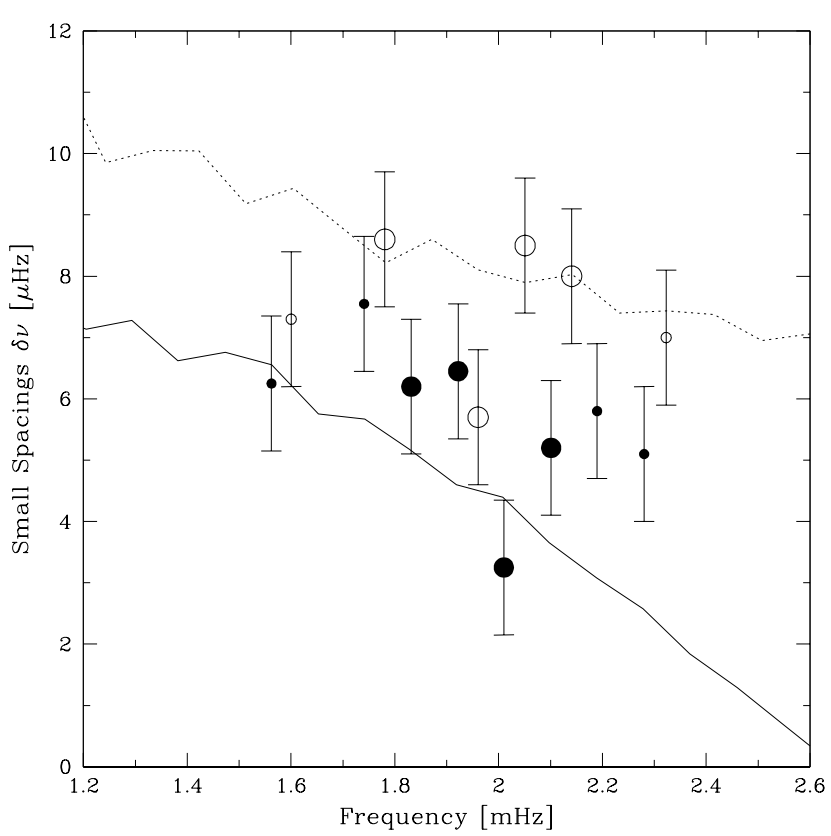

Fig. 6. Small separations for model OM. The full and open circles are the observed values obtained for $l=0-2$ and $l=1-3$. The small dots represent values involving modes detected at a low signal to noise ratio (see Paper I). The theoretical computations are represented as a solid curve for $l=0-2$ and a dotted curve for $l=1-3$.

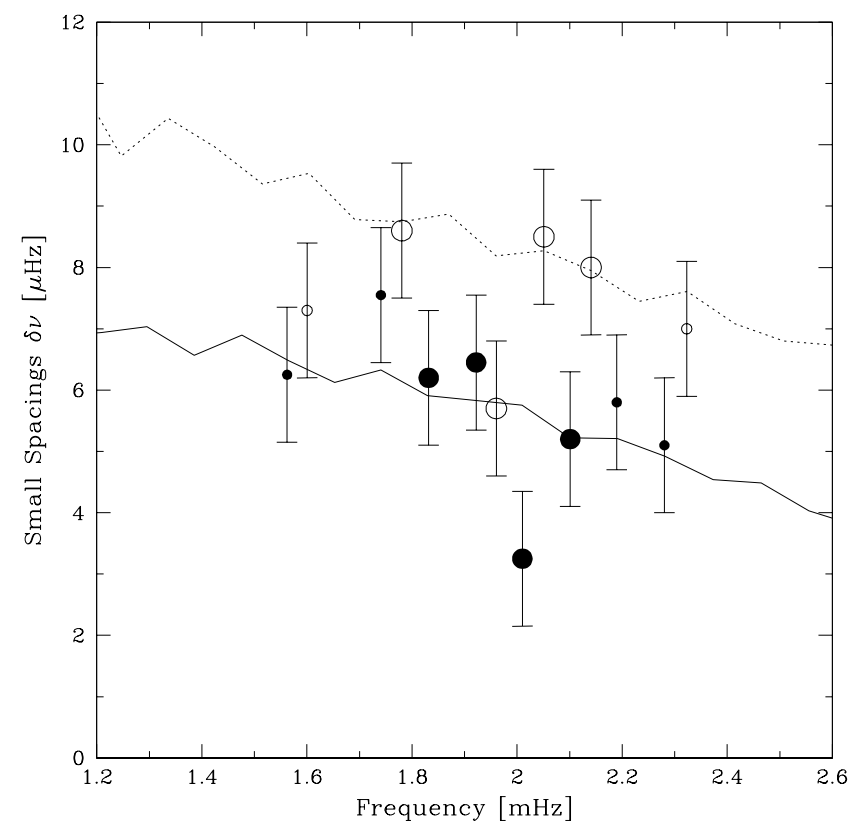

Fig. 7. Same as Fig. 6 for model AC.

highest possible signal-to-noise ratio, to be able to distinguish clearly between the two scenarios.

\section{Uncertainties}

Stellar models are characterized by their mass $(M)$, initial helium mass fraction $\left(Y_{0}\right)$, mixing-length parameter $(\alpha)$ and age.

Comparing Figs. 1 and 2 it is clear that the region of seismic constraint depends on the physics used in the model. The lines of constant average large separation are given for a set $\left(\alpha, Y_{0}\right)$.

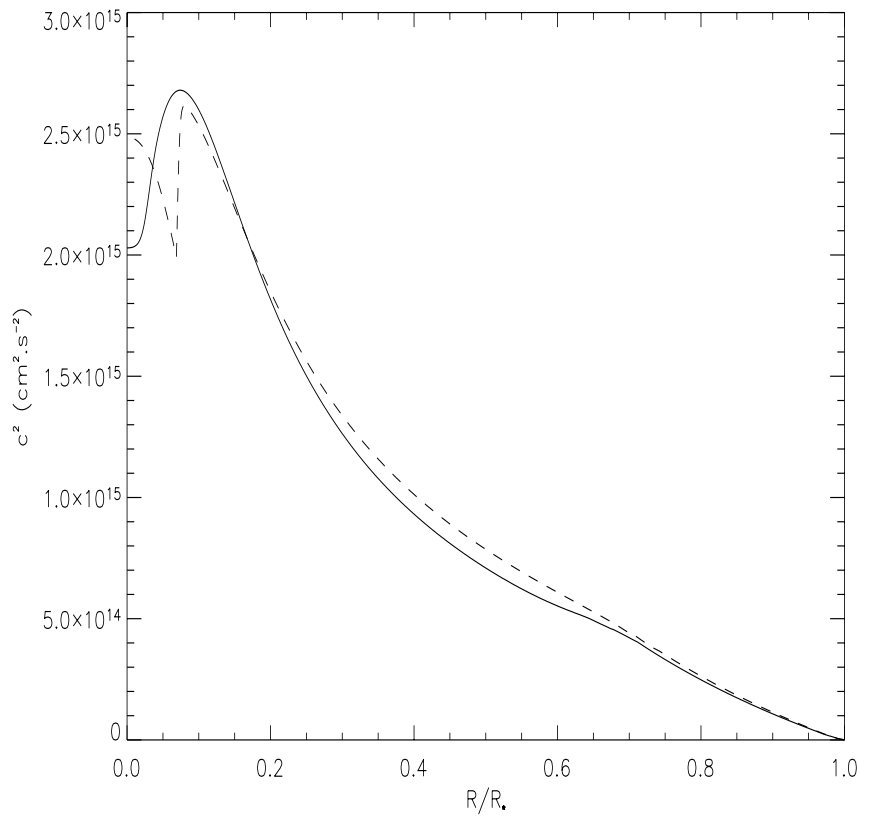

Fig. 8. Sound speed profiles inside AC (solid line) and OM (dashed line) models. The feature in the central region for the OM case compared to the AC case is due to the convective core. The sound velocity drops dramatically at its boundary and increases inside. The frequencies of the modes $l=0$ which progress down to the stellar center are affected by this behavior while those for which the turning point lies outside this region are not. This explains the behavior of the corresponding lines in the echelle diagram, also seen in the small separations.

Here $\alpha$ is adjusted to the solar models (Richard et al. 2004) and $Y_{0}$ is derived from the cosmic $Y-Z$ relation (Izotov \& Thuan 2001) Changes in $\alpha$ or $Y_{0}$ would also lead to modifications of the location of the iso- $\Delta v_{0}$ lines in the HR diagram. To estimate this effect, we have computed models with the same input parameters as $\mathrm{AC}$ or OM except that we imposed a small mixinglength or initial helium mass fraction variation. We then selected, for given physics (i.e. accretion or overmetallicity and a given set of parameters $\left.\left(\alpha, Y_{0}\right)\right)$, the age for which the average large separation is $90 \mu \mathrm{Hz}$.

Our results are given in Table 3 . The variations in $T_{\text {eff }}$ and $L / L_{\odot}$ are the differences between the values of the recomputed models and those of AC or OM. They are representative of the accuracy we can obtain with asteroseismology on $\alpha$ and $Y_{0}$. The changes we imposed on the mixing-length parameter and on the initial helium mass fraction correspond respectively to $3 \%$ and $4 \%$ of the initials values used in AC and OM. Variations in $Y_{0}$ with such an amplitude are ruled out because the resulting position of the star in the HR diagram would account no longer for the spectroscopic measurements. On the contrary a change in $\alpha$ of $3 \%$ has a very small influence on the star luminosity and does not affect the effective temperature as much as a variation in $Y_{0}$.

The problem is different for the precision obtained on the mass and the age of the star. In this case, a variation in these parameters will have no influence on the location of the iso$\Delta v_{0}$ lines (since neither $\alpha$ nor $Y_{0}$ vary). For given physics, 
Table 3. Difference of effective temperature and luminosity between sample models, AC or OM, and new models with $\Delta v_{0}=90 \mu \mathrm{Hz}$ but with a variation of either the initial helium fraction or the mixinglength parameter. The effective temperatures are given in $\mathrm{K}$.

\begin{tabular}{ccccccc}
\hline \hline Model & & \multicolumn{2}{c}{$\Delta \alpha$} & \multicolumn{2}{c}{$\Delta Y_{0}$} \\
\cline { 3 - 6 } \cline { 5 - 6 } & & -0.05 & +0.05 & & -0.01 & +0.01 \\
\hline \multirow{2}{*}{ AC } & $\Delta T_{\text {eff }}$ & -30.0 & +26.2 & -53.1 & +69.8 \\
& $\Delta\left(L / L_{\odot}\right)$ & -0.030 & +0.029 & -0.079 & +0.142 \\
\hline \multirow{2}{*}{ OM } & $\Delta T_{\text {eff }}$ & -7.3 & +9.7 & -57.4 & +66.3 \\
& $\Delta\left(L / L_{\odot}\right)$ & -0.009 & +0.016 & -0.073 & +0.084 \\
\hline
\end{tabular}

Table 4. Variation of the effective temperature, luminosity and average large separation for models AC and OM when subjects to a small variation of mass or age. The mass variation are given in $M_{\odot}$, the age in Myr the effective temperatures in $\mathrm{K}$ and the average large separation in $\mu \mathrm{Hz}$.

\begin{tabular}{|c|c|c|c|c|c|}
\hline \multirow[t]{2}{*}{ Model } & & \multicolumn{2}{|c|}{$\overline{\Delta M}$} & \multicolumn{2}{|c|}{$\Delta t$} \\
\hline & & $\overline{-0.01}$ & +0.01 & $\overline{-100}$ & $\overline{+100}$ \\
\hline \multirow{3}{*}{$\mathrm{AC}$} & $\Delta T_{\text {eff }}$ & +3.3 & -1.5 & +1.2 & +1.8 \\
\hline & $\Delta\left(L / L_{\odot}\right)$ & +0.003 & -0.003 & +0.007 & -0.006 \\
\hline & $\Delta\left(\Delta v_{0}\right)$ & -3.0 & +3.0 & +1.0 & -1.0 \\
\hline \multirow{3}{*}{$\mathrm{OM}$} & $\Delta T_{\text {eff }}$ & +8.0 & -3.1 & +11.1 & -11.1 \\
\hline & $\Delta\left(L / L_{\odot}\right)$ & -0.017 & +0.017 & -0.002 & +0.002 \\
\hline & $\Delta\left(\Delta v_{0}\right)$ & +3.0 & -2.0 & +1.0 & -1.0 \\
\hline
\end{tabular}

the knowledge of the effective temperature, the luminosity and the average large separation of the star helps to constrain values for its mass and its age. To estimate the precision obtained on both of these parameters, we computed models including the same physics but changing either the mass or the age. Table 4 shows the effect of a mass variation of $\pm 0.01 M_{\odot}$ or an age variation of $\pm 100 \mathrm{Myr}$ on $T_{\mathrm{eff}}, L / L_{\odot}$ and $\Delta v_{0}$.

This does not lead to major variations in effective temperature or in luminosity. In contrast, variations in $\Delta v_{0}$ are much more significant and do not agree with the seismical constraints in the case of a mass change of the order of $1 \%$. This gives an upper limit of $0.01 M_{\odot}$ for the precision obtained with asteroseismology on the mass of the star.

In case of an age shift of $\pm 100 \mathrm{Myr}$, the resulting models have average large separations corresponding to the limit values we used. Consequently, we expect, for given physics, an accuracy of the order of 100 Myr on the age of the star.

\section{Discussion and conclusions}

For the first time stellar oscillations have been detected and an asteroseismic analysis has been performed for a planet-hosting star. At magnitude $V=5.1, \mu$ Ara also represents the faintest solar-like star with detected p-mtdes. Forty three modes could be identified, leading to a clear ehelle diagram with large separations of $90 \mu \mathrm{Hz}$. We also discovered the smallest exoplanet ever observed, with a mass of 14 earth masses.
The seismic analysis of this star leads to precise constraints on its position in the HR diagram, inside the observational box obtained from spectroscopy and the Hipparcos parallax.

The aim of this run was to determine whether the star is completely overmetallic or only in its outer layers. In the first case it would suggest that the star and its planets formed out of an interstellar nebula which was already metal rich, while in the second case it would mean that accretion at the beginning of planet formation was much more active than generally thought.

We found a test to decide between these two fundamental scenarios: the overmetallic models which can account for the observable parameters of the star have a convective core while the accretion models do not. We chose two of these models for further analysis, by comparing the large and small separations. We found that the presence of a convective core in the overmetallic model has a signature: the lines $l=0$ and $l=2$ cross in the echelle diagram, which does not happen in the accretion model.

Unfortunately this signature clearly appears around frequencies of $2.5 \mathrm{mHz}$ which correspond to the highest frequency we were able to detect. Figures 6 and 7 seem in favor of the accretion scenario, but more observations are needed. It would be necessary to increase the signal to noise ratio for frequencies from 2.5 to $2.7 \mathrm{mHz}$, which lie in the crucial region where the small separations for the overmetallic and the accretion models clearly diverge.

In spite of a large number of observational constraints on the bright star $\mu$ Ara, we were not able to find a unique solution for the mass of the star and propose two possible masses which differ by more than $10 \%$. Further additional Doppler observations, from single or multi-sites, will not easily resolve the question. In the frequency range we are trying to reach, $p$-mode amplitudes are lower than $10 \mathrm{~cm} \mathrm{~s}^{-1}$ and due to their short life time, it will likely be very difficult to extract them from granulation noise. Future interferometric determinations of the radius of $\mu$ Ara could help in choosing one of the two scenarios, if it can reach an accuracy better than $1 \%$. Our analysis clearly illustrates the difficulties in characterizing the internal structure of stars even with a large number of identified $p$-modes.

Acknowledgements. The authors thank S. Charpinet and J. Christensen-Dalsgaard for their very constructive remarks. We are grateful to ESO staff support at the 3.6-m telescope. S.V. acknowledges a grant from Institut Universitaire de France. N.C.S. would like to thank the Swiss National Science Foundation and the Portuguese Fundação para Ciência e Tecnologia for the scolarship.

\section{References}

Angulo C., Arnould M., Rayet M., et al. 1999, Nucl. Phys. A 656, 1 Bazot, M., \& Vauclair, S. 2004, A\&A, 427, 965

Bensby, T., Feltzing, S., \& Lundström, I. 2003, A\&A, 410, 527

Bouchy, F., Bazot, M., Santos, N., et al. 2005, A\&A, 440, 609

Brassard, P., Pelletier, C., Fontaine, G., \& Wesemael, F. 1992, ApJS, 81,747

Favata, F., Micela, G., \& Sciortino, S. 1997, A\&A, 323, 809

Gabriel, M. 1989, A\&A, 226, 278

Gonzalez, G. 1998, A\&A, 334, 221

Iglesias, C. A., \& Rogers, F. J. 1996, ApJ, 464, 943 
Izotov, Y. I., \& Thuan, T. X. 2004, ApJ, 602, 200

Jones, H. R. A., Paul Butler, R., Marcy, G. W., et al. 2002, MNRAS, 337,1170

Laird, J. B. 1985, ApJS, 57, 389

Laws, C., Gonzalez, G., Walker, K. M., et al. 2003, AJ, 125, 2664

Mayor, M. \& Queloz, D. 1995, Nature, 378, 355

Michaud, G., Richard, O., Richer, J., \& VandenBerg, D. A. 2004, ApJ, 606, 452

Murray, N., \& Chaboyer, B. 2002, ApJ, 566, 442

Murray, N., Chaboyer, B., Arras, et al. 2001, ApJ, 555, 801

Paquette, C., Pelletier, C., Fontaine, G., \& Michaud, G. 1986, ApJS, 61,177
Perrin, M.-N., de Strobel, G. C., Cayrel, R., \& Hejlesen, P. M. 1977, A\&A, 54, 779

Richard, O., Théado, S., \& Vauclair, S. 2004, Sol. phys., in press Rogers, F. J., \& Nayfonov, A. 2002, ApJ 576, 1064

Roxburgh, I. W., \& Vorontsov, S. V. 1994, MNRAS, 267, 297

Santos, N. C., Israelian, G., Mayor, M., et al. 2003, A\&A, 398, 363

Santos, N. C., Israelian, G., \& Mayor, M. 2004a, A\&A, 415, 1153

Santos, N. C., Bouchy, F., Mayor, M., et al. 2004b, A\&A, 426, L19

Tassoul, M. 1980, ApJS, 43, 469

Trilling, D. E., Benz, W., Guillot, T., et al. 1998, ApJ, 500, 428

Udry, S., Mayor, M., \& Santos, N. C. 2003, A\&A, 407, 369 